\title{
RECHERCHES SUR L'ORIGINE DANS LE MIEL DU POLLEN DE PLANTES ENTOMOPHILES DÉPOURVUES DE NECTAIRES
}

PAR

\section{J. LOUVEAUX}

Station de Recherches apicoles, Bures-sur-Yvette.

Le spectre pollinique de nombreux miels fait ressortir la présence en proportion parfois élevée de grains de pollen appartenant à des plantes dépourvues de nectaires et que les abeilles ne visitent que pour la récolte du pollen. C'est le cas, par exemple, de Papaver Rhaeas dont on sait, de façon certaine, qu'il ne possède pas de nectaires mais dont le pollen est fréquent dans les miels.

On peut écarter de façon à peu près certaine l'hypothèse d'un transport par le vent en raison de la nature même du pollen de Papaver riche en gouttelettes grasses. Par contre il convient d'examiner sérieusement l'hypothèse d'une pollution accidentelle au cours des manipulations apicoles, l'apiculteur introduisant dans le miel au cours de son travail du pollen arraché aux cellules où il est habituellement stocké par les abeilles. On doit aussi retenir la possibilité d'une pollution opérée par les abeilles elles-mêmes et sans qu'interviennent les manipulations d'ordre apicole.

A fin de rechercher la valeur qu'il convient d'accorder à cette seconde hypothèse, nous avons organisé une expérience de butinage en serre selon le protocole que nous exposons ci-dessous.

Dans une serre de $9 \mathrm{~m}$ de long sur $3 \mathrm{~m}$ de large et $2,30 \mathrm{~m}$ de hauteur, très bien nettoyée et dábarrassée de toute végétation nous avons installé le 29 avril I957 une ruchette Dadant 3 cadres entièrement neuve et garnie seulement de cire gaufrée. Cette ruchette a été peuplée par secouage d'une petite colonie; on n'a donc introduit que des abeilles adultes en quantité suffisante pour couvrir les trois cadres. La reine était bien entendu présente.

Après une courte période de perturbation les abeilles s'habituent très bien à la vie en serre pourvu que la lumière soit suffisamment tamisée, ce que l'on obtient aisément par 1'emploi de verre cathédrale pour le vitrage et par la pose d'un clayonnage de bois continu appliqué à l'extérieur de 
toute la surface éclairante. Ce clayonnage arrête environ 50 à 60 p. Ioo de la lumière et la rend très diffuse.

Dès le lendemain on a commencé à nourrir la colonie au moxen d'un sirop composé de $5 \mathrm{~kg}$ de sucre pour 4 litres d'eau, soigneusement filtré sur coton de verre pour éliminer toutes les particules solides. I, nourrissement s'effectuait exclusivement à l'extérieur, la nourriture étant offerte dans unc série de boites de Pćtri garnies de billes de verre pour éviter les noyades. I e sirop était donné à volonté, les nourrisseurs étant maintenus constamment pleins. Toute la verrerie était nettoyée chaque jour à grande eau et résinfectée à l'eau de Javel.

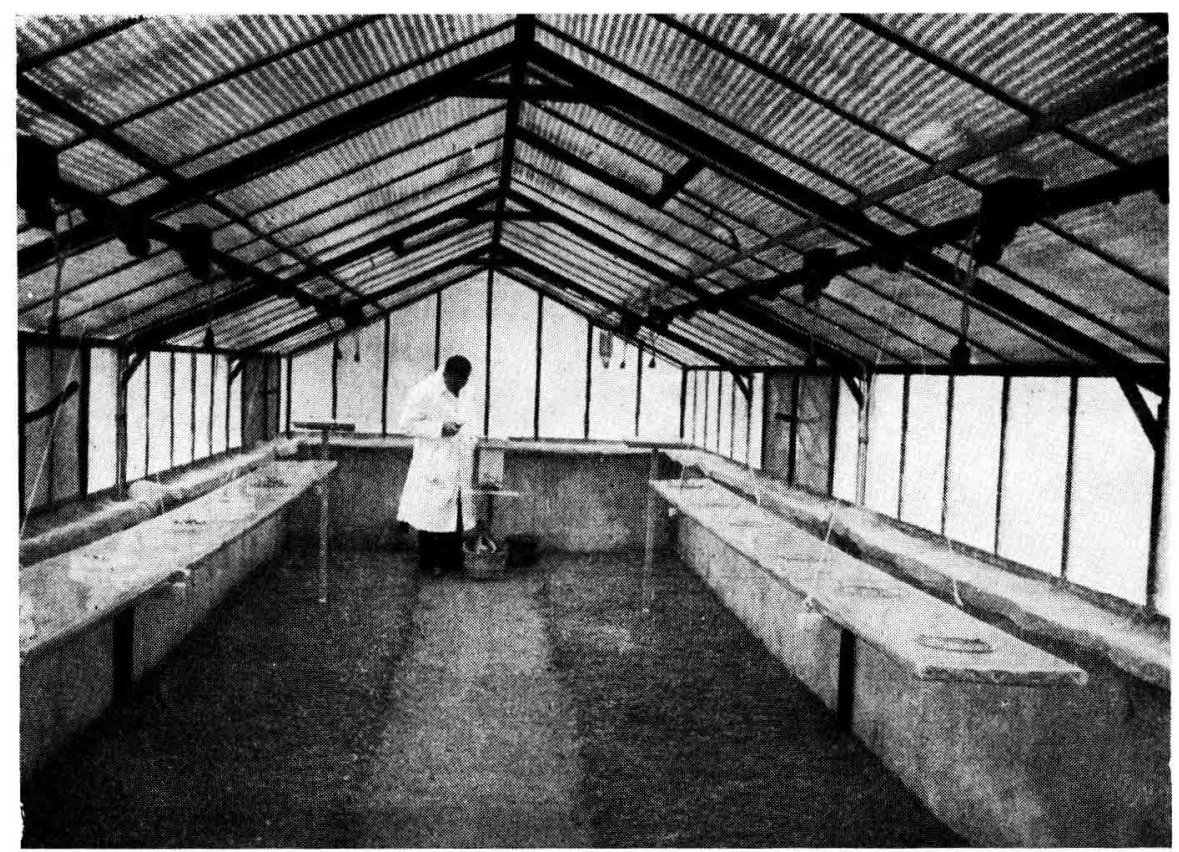

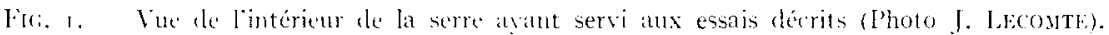

I a cire ganfrée est etirée sans délai par les abeilles qui commencent à déposer le sirop dans les cellules. I a ponte de la reine est constatée le I er mai. Fille ne cessera plus pendant toute la durée de l'expérience.

I.e 3 mai on installe les nourrisseurs à sirop d'un côté de la serre dans le sens de la longueur (voir fig. I) et, du cóté opposé c'est-à-dire à plus de deux mètres, on dispose 6 boites de P'étri contenant chacune une dizaine de grammes de pollen. Ce pollen est obtenu par broyage et tamisage de pelotes de pollen prélevées sur nos récoltes à la trappe à pollen. I1 provient surtout de Papaier Kaeas. Fin quelques heures les abeilles s'habituent à butiner sur les boites de P'étri et 1entrent à la ruche avec des, pelotes de taille normale. 
L'expérience s'est ainsi poursuivie jusqu'au 23 mai. Pendant cette période la ruchette a consommé ro $\mathrm{kg}$ de sucre environ et une quantité de pollen que l'on peut évaluer approximativement à $500 \mathrm{~g}$. En raison des pertes qui sont toujours importantes il est très difficile de chiffrer la quantité de pollen effectivement rentrée dans la ruchette.

Au cours des essais nous avons procédé à plusieurs reprises au marquage de butineuses, tant de sirop que de pollen. Ces marquages nous ont permis de mettre en évidence une très grande fidélité des abeilles à leur nourrisseur et surtout à leur type d'activité. Après plus de roo marquages nous n'avons retrouvé qu'une seule butineuse ayant changé d'activité et étant passée dans la même journée du butinage du pollen au butinage du sirop.

I e 2 I mai nous avons observé les premières naissances. L'extension du couvain était telle à la fin des essais que les trois cadres contenaient des larves sur les $2 / 3$ de leur surface environ, sauf une face d'un cadre latéral qui ne contenait que des réserves de miel et n'était pas encore complètement étiré. Il a d'ailleurs fallu transvaser la ruchette dans une ruche à 5 cadres dès le début de juin pour lui permettre de continuer à progresser. I a consommation, tant de sirop que de pollen, a toujours été croissante pendant le courant de mai.

Le 23 mai nous disposions sur l'un des cadres latéraux d'une surface importante de sirop operculé juste au-dessus du couvain. Nous avons prélevé ce cadre, brossé les abeilles et procédé au laboratoire à l'extraction du sirop. Les opercules étant enlevés au moyen d'un scalpel très propre nous avons vidé les cellules de leur contenu une par une à la trompe à vide. Nous avons ainsi obtenu $25^{\circ} \mathrm{g}$ d'un sirop doré, très visqueux, qui, en octobre ne présente encore aucun signe de cristallisation.

Nous avons soumis à l'analyse pollinique quantitative par la méthode de Matrizio (I) le sirop prélevé. Nous avons trouvé g ooo grains de pollen par ro g (moyenne de 4 mesures). I,e spectre pollinique du sirop est identique à celui du mélange donné en nourrissement, soit 80 p. roo de pollen de Papaver Rhaeas et 20 p. Ioo de Castanea, Plantago et Crucifères diverses. On constate que le " miel » est riche en petits débris divers dont beaucoup sont constitués par des fragments de grains de pollen; il contient quelques levures, quelques très rares spores de Cryptogames, très peu de bactéries.

On peut conclure de ces essais que, pollen et sirop étant récoltés séparément par des abeilles différentes on retrouve dans les provisions sucrées le pollen faisant la nourriture de base de la ruche. Cette constatation est capitale et permet d'expliquer pourquoi on retrouve le pollen de plantes sans nectaires dans le miel. I1 importe toutefois d'apporter quelques réserves quant à l'importance du mélange opéré par les abeilles. Si 1'on s'en rapporte au chiffre trouvé ( 9 ooo grains par Io g de miel) la 
pollution est forte. On peut se demander si les conditions artificielles de l'expérience n'ont pas contribué à déformer les chiffres. Il convient donc de tenir compte des points suivants :

Io Si l'abeille butineuse n'opère elle-même aucun mélange siroppollen, ce mélange doit être le fait des abeilles d'intérieur et notamment de celles, généralement jeunes, donc consommatrices de pollen, qui assurent la mise en place du miel dans les cellules. Selon l'importance des transferts qu'il aura subi le miel sera plus ou moins enrichi en pollen. Le rôle des jeunes abeilles consommatrices, donc vectrices de pollen, doit être prépondérant pour l'enrichissement du sirop en pollen. Le miel entreposé dans les hausses en période de grande miellée est probablement moins pollué que celui qui est déposé aux abords du couvain dans les conditions artificielles d'une serre où l'activité est assez réduite. Ce point reste à vérifier avec soin.

$2^{0}$ Malgré toutes les précautions prises il n'est pas exclu qu'une partie du pollen retrouvé dans le sirop provienne de déjections tombées accidentellement dans les nourrisseurs au cours de la journée. Nous noterons cependant que cette origine ne peut pas être très importante car nous n'avons tenu compte que des grains de pollen bien conformés, donc n'ayant subi aucun passage par l'intestin des abeilles.

Nous conclurons donc en disant que le miel peut sans aucun doute se trouver pollué à l'intérieur de la ruche et du seul fait des abeilles par des pollens n'appartenant pas aux gouttes de nectar mais que l'importance de cette pollution doit être très variable selon les circonstances.

De nombreux essais restent encore à réaliser dans ce domaine. Par l'expérimentation l'analyse pollinique des miels doit pouvoir trouver maintenant l'explication de phénomènes restés jusqu'ici obscurs.

\section{RÉFÉRENCES BIBLIOGRAPHIQUES}

(I) Maurizio (A.) . - Pollenanalytische Untersuchungen an Honig und Pollenhöschen, A. Beiträge zur quantitativen Pollenanalyse des Honigs. Schiciz. Bienen. Z., (Beihefte) 2, (I8), 320, 1949. 